\title{
Uniqueness and time oscillating behaviour of finite points blow-up solutions of the fast diffusion equation
}

\author{
Kin Ming Hui \\ Institute of Mathematics, Academia Sinica \\ Taipei, Taiwan, R. O. C.
}

May 26, 2018

\begin{abstract}
Let $n \geq 3$ and $0<m<\frac{n-2}{n}$. We will extend the results of J.L. Vazquez and M. Winkler [VW2] and prove the uniqueness of finite points blow-up solutions of the fast diffusion equation $u_{t}=\Delta u^{m}$ in both bounded domains and $\mathbb{R}^{n} \times(0, \infty)$. We will also construct initial data such that the corresponding solution of the fast diffusion equation in bounded domain oscillate between infinity and some positive constant as $t \rightarrow \infty$.
\end{abstract}

Key words: uniqueness, fast diffusion equation, time oscillating behaviour AMS 2010 Mathematics Subject Classification: Primary 35K65 Secondary 35B51, 35B40

\section{Introduction}

The equation

$$
u_{t}=\Delta u^{m}
$$

arises in many physical and geometrical models [A], [DK], [V1], [V2]. When $m>1,(1.1)$ is called porous medium equation which appears in the modeling of the flow of gases through porous media and oil passing through sand etc. (1.1) also arises as the large time asymptotic limit solution of the compressible Euler equation with damping [HPW], [LZ]. When $m=1$, (1.1) is the heat equation. When $0<m<1$, (1.1) is called the fast diffusion equation. When $m=\frac{n-2}{n+2}$ and $n \geq 3$, (1.1) arises in the study of Yamabe flow on $\mathbb{R}^{n}$ [DS1], [DS2], [DS]. Note that the metric $g_{i j}=u^{\frac{4}{n+2}} d x^{2}, u>0, n \geq 3$, is a solution of the Yamabe flow [DS2], [DS],

$$
\frac{\partial g_{i j}}{\partial t}=-R g_{i j} \quad \text { in } \mathbb{R}^{n} \times(0, T)
$$


if and only if $u$ is a solution of

$$
u_{t}=\frac{n-1}{m} \Delta u^{m}
$$

in $\mathbb{R}^{n} \times(0, T)$ with $m=\frac{n-2}{n+2}$ where $R(\cdot, t)$ is the scalar curvature of the metric $g_{i j}(\cdot, t)$. Recently F. Golse and F. Salvarani [GS], B. Choi and K. Lee [CL], have shown that (1.1) also appears as the nonlinear diffusion limit for the generalized Carleman models.

Although there are a lot of study of (1.1) for the case $m>\frac{(n-2)_{+}}{n}$, there are not many results of (1.1) for the case $0<m<\frac{n-2}{n}, n \geq 3$. When $0<m \leq \frac{n-2}{n}$ and $n \geq 3$, existence of positive smooth solutions of

$$
\left\{\begin{aligned}
u_{t} & =\Delta u^{m}, u \geq 0, & & \text { in } \mathbb{R}^{n} \times(0, T) \\
u(x, 0) & =u_{0} & & \text { in } \mathbb{R}^{n}
\end{aligned}\right.
$$

for any $0 \leq u_{0} \in L_{l o c}^{p}\left(\mathbb{R}^{n}\right), p>(1-m) n / 2$, satisfying the condition,

$$
\liminf _{R \rightarrow \infty} \frac{1}{R^{n-\frac{2}{1-m}}} \int_{|x| \leq R} u_{0} d x \geq C_{1} T^{\frac{1}{1-m}}
$$

for some constant $C_{1}>0$ is proved by S.Y. Hsu in [Hs].

Let $\Omega \subset \mathbb{R}^{n}$ be a smooth bounded domain. When $0<m \leq \frac{n-2}{n}, n \geq 3$ and $0 \in \Omega$, existence of singular solutions and asymptotic large time behaviour of $(1.1)$ in $(\Omega \backslash\{0\}) \times$ $(0, \infty)$ which blows up at $\{0\} \times(0, \infty)$ when the initial value $u_{0}$ satisfies

$$
c_{1}|x|^{-\gamma_{1}} \leq u_{0}(x) \leq c_{2}|x|^{-\gamma_{2}} \quad \forall x \in \Omega \backslash\{0\}
$$

for some constants $c_{1}>0, c_{2}>0$ and $\gamma_{2} \geq \gamma_{1}>\frac{2}{1-m}$ were proved by J.L. Vazquez and M. Winkler in [VW1]. Uniqueness of singular solutions of [1.1) in $(\Omega \backslash\{0\}) \times(0, \infty)$ that blows up at $\{0\} \times(0, \infty)$ and existence of singular initial data such that the corresponding singular solution of (1.1) in $(\Omega \backslash\{0\}) \times(0, \infty)$ oscillates between infinity and some positive constant as $t \rightarrow \infty$ are proved by J.L. Vazquez and M. Winkler in [VW2].

When $0<m \leq \frac{n-2}{n}$ and $n \geq 3$, existence of singular solutions of (1.1) in $\left(\mathbb{R}^{n} \backslash\{0\}\right) \times(0, \infty)$ which blows up at $\{0\} \times(0, \infty)$ when the initial value $u_{0}$ satisfies

$$
c_{1}|x|^{-\gamma} \leq u_{0}(x) \leq c_{2}|x|^{-\gamma} \quad \forall x \in \mathbb{R}^{n} \backslash\{0\}
$$

for some constants $c_{1}>0, c_{2}>0$ and $\frac{2}{1-m}<\gamma<\frac{n-2}{m}$ was proved by K. M. Hui and Soojung $\mathrm{Kim}$ in [HKs]. Asymptotic large time behaviour of such solution was also proved by K. M. Hui and Soojung Kim in [HKs] when $\frac{2}{1-m}<\gamma<n$.

Let $a_{1}, a_{2}, \ldots, a_{i_{0}} \in \Omega, \widehat{\Omega}=\Omega \backslash\left\{a_{1}, a_{2}, \ldots, a_{i_{0}}\right\}$ and $\widehat{\mathbb{R}^{n}}=\mathbb{R}^{n} \backslash\left\{a_{1}, a_{2}, \ldots, a_{i_{0}}\right\}$. For any $\delta>0$, let $\Omega_{\delta}=\Omega \backslash\left(\cup_{i=1}^{i_{0}} B_{\delta}\left(a_{i}\right)\right)$ and $\mathbb{R}_{\delta}^{n}=\mathbb{R}^{n} \backslash\left(\cup_{i=1}^{i_{0}} B_{\delta}\left(a_{i}\right)\right)$ where $B_{R}\left(x_{0}\right)=\left\{x \in \mathbb{R}^{n}:\left|x-x_{0}\right|<R\right\}$, $B_{R}=B_{R}(0), \widehat{B}_{R}\left(x_{0}\right)=B_{R}\left(x_{0}\right) \backslash\left\{x_{0}\right\}$ and $\widehat{B}_{R}=\widehat{B}_{R}(0)$ for any $x_{0} \in \mathbb{R}^{n}$ and $R>0$. Let $\delta_{0}(\Omega)=$ $\frac{1}{3} \min _{1 \leq i, j \leq i_{0}}\left(\operatorname{dist}\left(a_{i}, \Omega\right),\left|a_{i}-a_{j}\right|\right)$ and $\delta_{0}\left(\mathbb{R}^{n}\right)=\frac{1}{3} \min _{1 \leq i, j \leq i_{0}}\left|a_{i}-a_{j}\right|$. For any $0<\delta \leq \delta_{0}(\Omega)$, let $D_{\delta}=\{x \in \Omega$ : $\operatorname{dist}(x, \partial \Omega)<\delta\}$. Let $R_{0}>0$ be such that $a_{1}, \cdots, a_{i_{0}} \in B_{R_{0}}$. For any $R>R_{0}$ 
and $0<\delta \leq \delta_{0}\left(\mathbb{R}^{n}\right)$, let $\Omega_{\delta, R}=B_{R} \backslash \cup_{i=1}^{i_{0}} B_{\delta}\left(a_{i}\right)$. When there is no ambiguity we will drop the parameter and write $\delta_{0}$ instead of $\delta_{0}(\Omega)$ or $\delta_{0}\left(\mathbb{R}^{n}\right)$. Unless stated otherwise we will assume that $0<m<\frac{n-2}{n}$ and $n \geq 3$ for the rest of the paper.

Existence of singular solutions of (1.1) in $\widehat{\Omega} \times(0, T)$ which blows up at $\left\{a_{1}, a_{2}, \ldots, a_{i_{0}}\right\} \times$ $(0, T)$ was proved by K.M. Hui and Sunghoon Kim in [HK1] when the initial value $u_{0}$ satisfies

$$
u_{0}(x) \approx\left|x-a_{i}\right|^{-\gamma_{i}} \quad \text { for } x \approx a_{i} \quad \forall i=1,2, \ldots, i_{0}
$$

for some constants $\gamma_{i}>\max \left(\frac{n}{2 m}, \frac{n-2}{m}\right)$ for any $i=1,2, \ldots, i_{0}$. When $0 \leq f \in L^{\infty}(\partial \Omega \times[0, \infty))$ and the initial value $0 \leq u_{0} \in L_{l o c}^{p}\left(\bar{\Omega} \backslash\left\{a_{1}, \cdots, a_{i_{0}}\right\}\right)\left(L_{l o c}^{p}\left(\widehat{\mathbb{R}^{n}}\right)\right.$ respectively) for some constant $p>\frac{n(1-m)}{2}$ satisfies

$$
u_{0}(x) \geq \frac{\lambda_{i}}{\left|x-a_{i}\right|^{\gamma_{i}}} \quad \forall 0<\left|x-a_{i}\right|<\delta_{1}, i=1, \cdots, i_{0}
$$

for some constants $0<\delta_{1}<\min \left(1, \delta_{0}\right), \lambda_{1}, \cdots, \lambda_{i_{0}} \in \mathbb{R}^{+}$and $\gamma_{1}, \cdots, \gamma_{i_{0}} \in\left(\frac{2}{1-m}, \infty\right)$, existence of singular solutions of

$$
\left\{\begin{aligned}
u_{t} & =\Delta u^{m} & & \text { in } \widehat{\Omega} \times(0, \infty) \\
u & =f & & \text { on } \partial \Omega \times(0, \infty) \\
u\left(a_{i}, t\right) & =\infty & & \forall t>0, i=1, \cdots, i_{0} \\
u(x, 0) & =u_{0}(x) & & \text { in } \widehat{\Omega}
\end{aligned}\right.
$$

and

$$
\left\{\begin{aligned}
u_{t} & =\Delta u^{m} & & \text { in } \widehat{\mathbb{R}^{n}} \times(0, \infty) \\
u\left(a_{i}, t\right) & =\infty & & \forall i=1, \cdots, i_{0}, t>0 \\
u(x, 0) & =u_{0}(x) & & \text { in } \widehat{\mathbb{R}^{n}}
\end{aligned}\right.
$$

respectively were proved by K.M. Hui and Sunghoon Kim in [HK2]. It was proved in [HK2] that the singular solutions of (1.3) and (1.4) constructed in [HK2] have the property that for any $T>0$ and $\delta_{2} \in\left(0, \delta_{1}\right)$ there exists a constant $C_{1}>0$ such that

$$
u(x, t) \geq \frac{C_{1}}{\left|x-a_{i}\right|^{\gamma_{i}}} \quad \forall 0<\left|x-a_{i}\right|<\delta_{2}, 0<t<T, i=1,2, \cdots, i_{0} .
$$

Moreover [HK2] if the initial value $u_{0}$ also satisfies

$$
u_{0}(x) \leq \frac{\lambda_{i}^{\prime}}{\left|x-a_{i}\right|^{\gamma_{i}^{\prime}}} \quad \forall 0<\left|x-a_{i}\right|<\delta_{1}, i=1, \cdots, i_{0},
$$

for some constants $\lambda_{1}^{\prime}, \cdots, \lambda_{i_{0}}^{\prime} \in \mathbb{R}^{+}$, and $\gamma_{i}^{\prime} \geq \gamma_{i}$ for all $i=1, \cdots, i_{0}$, then for any $T>0$ and $\delta_{2} \in\left(0, \delta_{1}\right)$ there exists a constant $C_{2}>0$ such that the singular solutions of (1.3) and (1.4) constructed in [HK2] satisfies

$$
u(x, t) \leq \frac{C_{2}}{\left|x-a_{i}\right|_{i}^{\prime}} \quad \forall 0<\left|x-a_{i}\right|<\delta_{2}, 0<t<T, i=1,2, \cdots, i_{0} .
$$


When $f \geq \mu_{0}$ and $u_{0} \geq \mu_{0}$ for some constant $\mu_{0}>0$, the singular solutions of (1.3) and (1.4) constructed in [HK2] also satisfy

$$
u(x, t) \geq \mu_{0} \quad \forall x \in \widehat{\Omega}\left(\widehat{\mathbb{R}^{n}} \text { respectively }\right), t>0 .
$$

Asymptotic large time behaviour of such singular solutions was also proved by K.M. Hui and Sunghoon Kim in [HK2].

In this paper we will extend the results of J.L. Vazquez and M. Winkler [VW2] and prove the uniqueness of singular solutions of (1.3) and (1.4). We will also construct initial data $u_{0}$ such that the corresponding singular solution of (1.3) with $f=\mu_{0}>0$ oscillates between infinity and some positive constant as $t \rightarrow \infty$. More precisely we will prove the following results.

Theorem 1.1. Let $n \geq 3,0<m<\frac{n-2}{n}, 0<\delta_{1}<\min \left(1, \delta_{0}\right), \mu_{0}>0, f_{1}, f_{2} \in C^{3}(\partial \Omega \times(0, \infty)) \cap$ $L^{\infty}(\partial \Omega \times(0, \infty))$ be such that $f_{2} \geq f_{1} \geq \mu_{0}$ on $\partial \Omega \times(0, \infty)$ and

$$
\mu_{0} \leq u_{0,1} \leq u_{0,2} \in L_{l o c}^{p}\left(\bar{\Omega} \backslash\left\{a_{1}, \cdots, a_{i_{0}}\right\}\right) \quad \text { for some constant } p>\frac{n(1-m)}{2}
$$

be such that

$$
\frac{\lambda_{i}}{\left|x-a_{i}\right|^{\gamma_{i}}} \leq u_{0,1}(x) \leq u_{0,2} \leq \frac{\lambda_{i}^{\prime}}{\left|x-a_{i}\right|^{\gamma_{i}^{\prime}}} \quad \forall 0<\left|x-a_{i}\right|<\delta_{1}, i=1, \cdots, i_{0}
$$

holds for some constants $\lambda_{1}, \cdots, \lambda_{i_{0}}, \lambda_{1}^{\prime}, \cdots, \lambda_{i_{0}}^{\prime} \in \mathbb{R}^{+}$and

$$
\gamma_{i}^{\prime} \geq \gamma_{i}>\frac{2}{1-m} \quad \forall i=1,2, \ldots, i_{0} .
$$

Suppose $u_{1}, u_{2}$, are the solutions of (1.3) with $u_{0}=u_{0,1}, u_{0,2}, f=f_{1}, f_{2}$, respectively which satisfies

$$
u_{j}(x, t) \geq \mu_{0} \quad \forall x \in \widehat{\Omega}, t>0, j=1,2
$$

such that for any constants $T>0$ and $\delta_{2} \in\left(0, \delta_{1}\right)$ there exist constants $C_{1}=C_{1}(T)>0$, $C_{2}=C_{2}(T)>0$, such that

$$
\frac{C_{1}}{\left|x-a_{i}\right|^{\gamma_{i}}} \leq u_{j}(x, t) \leq \frac{C_{2}}{\left|x-a_{i}\right|^{\gamma_{i}^{\prime}}} \quad \forall 0<\left|x-a_{i}\right|<\delta_{2}, 0<t<T, i=1,2, \ldots, i_{0}, j=1,2 .
$$

Suppose $u_{1}, u_{2}$, also satisfies

$$
\left\|u_{i}(\cdot, t)-u_{0, i}\right\|_{L^{1}\left(\Omega_{\delta}\right)} \rightarrow 0 \quad \text { as } t \rightarrow 0 \quad \forall 0<\delta<\delta_{0}, i=1,2 .
$$

Then

$$
u_{1}(x, t) \leq u_{2}(x, t) \quad \forall x \in \widehat{\Omega}, t>0 .
$$


Theorem 1.2. Let $n \geq 3,0<m<\frac{n-2}{n}, 0<\delta_{1}<\min \left(1, \delta_{0}\right), \mu_{0}>0, \mu_{0} \leq f_{1} \leq f_{2} \in$ $L^{\infty}(\partial \Omega \times(0, \infty))$ and (1.9), (1.10), hold for some constants $\lambda_{1}, \cdots, \lambda_{i_{0}}, \lambda_{1}^{\prime}, \cdots, \lambda_{i_{0}}^{\prime} \in \mathbb{R}^{+}$and

$$
\frac{2}{1-m}<\gamma_{i} \leq \gamma_{i}^{\prime}<\frac{n-2}{m} \quad \forall i=1,2, \ldots, i_{0} .
$$

Suppose $u_{1}, u_{2}$, are the solutions of (1.3) with $u_{0}=u_{0,1}, u_{0,2}, f=f_{1}, f_{2}$, respectively which satisfies (1.12) and (1.14) such that for any constants $T>0$ and $\delta_{2} \in\left(0, \delta_{1}\right)$ there exist constants $C_{1}=C_{1}(T)>0, C_{2}=C_{2}(T)>0$, such that (1.13) holds. Then (1.15) holds.

Theorem 1.3. Let $n \geq 3,0<m<\frac{n-2}{n}, 0<\delta_{1}<\min \left(1, \delta_{0}\right), \mu_{0}>0, R_{1}>R_{0}$ and $\mu_{0} \leq u_{0,1} \leq$ $u_{0,2} \in L_{l o c}^{p}\left(\widehat{\mathbb{R}^{n}}\right)$ for some constant $p>\frac{n(1-m)}{2}$ such that

$$
\int_{\mathbb{R}^{n} \backslash B_{R_{1}}}\left|u_{0, j}-\mu_{0}\right| d x<\infty \quad \forall j=1,2 .
$$

Let (1.10) hold for some constants $\lambda_{1}, \cdots, \lambda_{i_{0}}, \lambda_{1}^{\prime}, \cdots, \lambda_{i_{0}}^{\prime} \in \mathbb{R}^{+}$and

$$
\frac{2}{1-m}<\gamma_{i} \leq \gamma_{i}^{\prime}<n \quad \forall i=1,2, \ldots, i_{0}
$$

Suppose $u_{1}, u_{2}$, are the solutions of (1.4) with $u_{0}=u_{0,1}, u_{0,2}$ respectively which satisfy

$$
u_{j}(x, t) \geq \mu_{0} \quad \forall x \in \widehat{\mathbb{R}^{n}}, t>0, j=1,2
$$

and

$$
\int_{\widehat{\mathbb{R}^{n}}}\left|u_{j}(x, t)-\mu_{0}\right| d x \leq \int_{\widehat{\mathbb{R}}^{n}}\left|u_{0, j}-\mu_{0}\right| d x \quad \forall t>0, j=1,2
$$

such that for any constants $T>0$ and $\delta_{2} \in\left(0, \delta_{1}\right)$ there exist constants $C_{1}=C_{1}(T)>0$, $C_{2}=C_{2}(T)>0$, such that (1.13) holds. Then

$$
u_{1}(x, t) \leq u_{2}(x, t) \quad \forall x \in \widehat{\mathbb{R}^{n}}, t>0
$$

Theorem 1.4. Let $n \geq 3,0<m<\frac{n-2}{n}, 0<\delta_{1}<\min \left(1, \delta_{0}\right)$ and $\mu_{0}>0$. There there exists $u_{0} \in L_{l o c}^{p}\left(\bar{\Omega} \backslash\left\{a_{1}, \cdots, a_{i_{0}}\right\}\right)$ for some constant $p>\frac{n(1-m)}{2}, u_{0} \geq \mu_{0}$ in $\widehat{\Omega}$, such that

$$
\frac{\lambda_{i}}{\left|x-a_{i}\right|^{\gamma_{i}}} \leq u_{0}(x) \leq \frac{\lambda_{i}^{\prime}}{\left|x-a_{i}\right|^{\gamma_{i}^{\prime}}} \quad \forall 0<\left|x-a_{i}\right|<\delta_{1}, i=1, \cdots, i_{0}
$$

for some constants satisfying (1.11) and $\lambda_{1}, \cdots, \lambda_{i_{0}}, \lambda_{1^{\prime}}^{\prime} \cdots, \lambda_{i_{0}}^{\prime} \in \mathbb{R}^{+}$such that

$$
\left\{\begin{aligned}
u_{t} & =\Delta u^{m} & & \text { in } \widehat{\Omega} \times(0, \infty) \\
u & =\mu_{0} & & \text { on } \partial \Omega \times(0, \infty) \\
u\left(a_{i}, t\right) & =\infty & & \forall t>0, i=1, \cdots, i_{0} \\
u(x, 0) & =u_{0}(x) & & \text { in } \widehat{\Omega}
\end{aligned}\right.
$$

has a unique solution $u$ with the property that $u$ oscillates between $\mu_{0}$ and infinity as $t \rightarrow \infty$. 
The plan of the paper is as follows. In section 2 we will prove the uniqueness of singular solutions of (1.3) and (1.4). In section 3 we will prove the existence of initial data such that the corresponding solution of (1.3) with $f=\mu_{0}>0$ oscillates between infinity and some positive constant as $t \rightarrow \infty$.

We start with some definitions. For any $0 \leq f \in L^{\infty}(\partial \Omega \times(0, \infty))$ and $0 \leq u_{0} \in L_{l o c}^{1}(\widehat{\Omega})$, we say that $u$ is a solution of

$$
\left\{\begin{aligned}
u_{t} & =\Delta u^{m} & & \text { in } \widehat{\Omega} \times(0, \infty) \\
u & =f & & \text { on } \partial \Omega \times(0, \infty) \\
u(x, 0) & =u_{0}(x) & & \text { in } \widehat{\Omega}
\end{aligned}\right.
$$

if $\left.u \in L_{\text {loc }}^{\infty} \bar{\Omega} \backslash\left\{a_{1}, \cdots, i_{0}\right\} \times(0, \infty)\right)$ is positive in $\widehat{\Omega} \times(0, \infty)$ and satisfies (1.1) in $\widehat{\Omega} \times(0, \infty)$ in the classical sense with

for any compact set $K \subset \widehat{\Omega}$ and

$$
\left\|u(\cdot, t)-u_{0}\right\|_{L^{1}(K)} \rightarrow 0 \quad \text { as } t \rightarrow 0
$$

$$
\begin{aligned}
& \int_{t_{1}}^{t_{2}} \int_{\widehat{\Omega}}\left(u \eta_{t}+u^{m} \Delta \eta\right) d x d t \\
& \quad=\int_{t_{1}}^{t_{2}} \int_{\partial \Omega} f^{m} \frac{\partial \eta}{\partial \nu} d \sigma d t+\int_{\widehat{\Omega}} u\left(x, t_{2}\right) \eta\left(x, t_{2}\right) d x-\int_{\widehat{\Omega}} u\left(x, t_{1}\right) \eta\left(x, t_{1}\right) d x
\end{aligned}
$$

for any $t_{2}>t_{1}>0$ and $\eta \in C_{c}^{2}\left(\left(\bar{\Omega} \backslash\left\{a_{1}, \cdots, a_{i_{0}}\right\}\right) \times(0, \infty)\right)$ satisfying $\eta \equiv 0$ on $\partial \Omega \times(0, T)$. We say that $u$ is a solution of (1.3) if $u$ is a solution of (1.24) and satisfies

$$
u(x, t) \rightarrow \infty \quad \text { as } x \rightarrow a_{i} \quad \forall t>0, i=1, \cdots, i_{0} .
$$

For any $0 \leq u_{0} \in L_{l o c}^{1}\left(\widehat{\mathbb{R}^{n}}\right)$ we say that $u$ is a solution of $(1.4)$ if $u \in L_{l o c}^{\infty}\left(\widehat{\mathbb{R}^{n}} \times(0, \infty)\right)$ is positive in $\widehat{\mathbb{R}^{n}} \times(0, \infty)$ and satisfies (1.1) in $\widehat{\mathbb{R}^{n}} \times(0, \infty)$ in the classical sense and (1.25), (1.27), hold for any compact set $K \subset \widehat{\mathbb{R}^{n}}$.

For any set $A \subset \mathbb{R}^{n}$, we let $\chi_{A}$ be the characteristic function of the set $A$. For any $a \in \mathbb{R}$, we let $a_{+}=\max (0, a)$.

\section{Uniqueness of solution}

In this section we will prove the uniqueness of singular solutions of (1.3) and (1.4).

Lemma 2.1. Let $n \geq 3,0<m<\frac{n-2}{n}, 0<\delta_{1}<\delta_{0}, 0 \leq f \in L^{\infty}(\partial \Omega \times[0, \infty))$ and $0 \leq u_{0} \in$ $L_{\text {loc }}^{p}\left(\bar{\Omega} \backslash\left\{a_{1}, \cdots, a_{i_{0}}\right\}\right)$ for some constant $p>\frac{n(1-m)}{2}$ be such that (1.2) holds for some constants $\lambda_{1}$, $\cdots, \lambda_{i_{0}} \in \mathbb{R}^{+}$and $\gamma_{1}, \cdots, \gamma_{i_{0}} \in\left(\frac{2}{1-m}, \infty\right)$. Let $u$ be the solution of (1.3) constructed in Theorem 1.1 of HK2]. Then there exists a constant $C>0$ such that

$$
\int_{D_{\delta}} u(x, t) d x \leq\left\{\left(\int_{D_{2 \delta}} u_{0} d x\right)^{1-m}+C t\right\}^{\frac{1}{1-m}}+\mid D_{\delta}\|f\|_{L^{\infty}} \quad \forall t>0,0<\delta<\delta_{0} / 2
$$


holds.

Proof. We will use a modification of the proof of Theorem 2.2 of [HP] to prove this lemma. For any $0<\varepsilon<1$, let $k>\|f\|_{L^{\infty}}+\varepsilon$. Let $0<\delta<\delta_{0} / 2$. We choose $\phi \in C^{\infty}(\bar{\Omega})$ such that $0 \leq \phi \leq 1$ in $\bar{\Omega}, \phi(x)=0$ for any $x \in \Omega \backslash D_{2 \delta}$ and $\phi(x)=1$ for any $x \in \overline{D_{\delta}}$. Let $\alpha>\frac{2}{1-m}$ and $\eta(x)=\phi(x)^{\alpha}$. Then by direct computation,

$$
C_{\eta}:=\left(\int_{\Omega}\left(\eta^{-m}|\Delta \eta|\right)^{\frac{1}{1-m}} d x\right)^{1-m}<\infty .
$$

For any $0<\varepsilon<1$ and $M>0$, let

$$
\left\{\begin{aligned}
u_{0, M}(x) & =\min \left(u_{0}(x), M\right) \\
u_{0, \varepsilon, M}(x) & =\min \left(u_{0}(x), M\right)+\varepsilon \\
f_{\varepsilon}(x, t) & =f(x, t)+\varepsilon \quad \forall(x, t) \in \partial \Omega \times(0, \infty)
\end{aligned}\right.
$$

and let $u_{M}$ and $u_{\varepsilon, M}$ be the solutions of

$$
\left\{\begin{aligned}
u_{t} & =\Delta u^{m} & & \text { in } \Omega \times(0, \infty) \\
u(x, t) & =f_{\varepsilon} & & \text { on } \partial \Omega \times(0, \infty) \\
u(x, 0) & =u_{0, \varepsilon, M} & & \text { in } \Omega .
\end{aligned}\right.
$$

and

$$
\left\{\begin{aligned}
u_{t} & =\Delta u^{m} & & \text { in } \Omega \times\left(0, T_{M}\right) \\
u(x, t) & =f & & \text { on } \partial \Omega \times\left(0, T_{M}\right) \\
u(x, 0) & =u_{0, M} & & \text { in } \Omega
\end{aligned}\right.
$$

respectively constructed in [HK2] for some maximal time $T_{M}>0$ of existence. By the result in [HK2] $T_{M} \rightarrow \infty$ as $M \rightarrow \infty$. Moreover $u_{\varepsilon, M}$ decreases and converges to $u_{M}$ in $\Omega \times\left(0, T_{M}\right)$ uniformly in $C^{2,1}(K)$ for every compact subset $K$ of $\Omega$ as $\varepsilon \rightarrow 0$ and $u_{M}$ increases and converges to $u$ in $\widehat{\Omega} \times(0, \infty)$ uniformly in $C^{2,1}(K)$ for every compact subset $K$ of $\widehat{\Omega}$ as $M \rightarrow \infty$. By approximation we may assume without loss of generality that $u_{\varepsilon, M} \in C^{2}(\bar{\Omega} \times(0, \infty))$. Then by the Kato inequality $([\overline{\mathrm{DK}}],[\mathrm{K}])$,

$$
\begin{aligned}
\frac{\partial}{\partial t}\left(\int_{\Omega}\left(u_{\varepsilon, M}-k\right)_{+} \eta d x\right) & \leq \int_{\Omega}\left(u_{\varepsilon, M}^{m}-k^{m}\right)_{+} \Delta \eta d x \\
& \leq C \int_{\Omega}\left(u_{\varepsilon, M}-k\right)_{+}^{m}|\Delta \eta| d x \\
& \leq C\left(\int_{\Omega}\left(u_{\varepsilon, M}-k\right)_{+} \eta d x\right)^{m}\left(\int_{\Omega}\left(\eta^{-m}|\Delta \eta|\right)^{\frac{1}{1-m}} d x\right)^{1-m} \\
& =C_{1}\left(\int_{\Omega}\left(u_{\varepsilon, M}-k\right)_{+} \eta d x\right)^{m} \quad \forall t>0,0<\varepsilon<1, M>0
\end{aligned}
$$


for some constants $C>0, C_{1}>0$. Integrating (2.2) over $(0, t)$ and letting $\varepsilon \rightarrow 0, M \rightarrow \infty$ and $k \rightarrow\|f\|_{L^{\infty}}$,

$$
\int_{D_{\delta}}\left(u(x, t)-\|f\|_{L^{\infty}}\right)_{+} d x \leq\left\{\left(\int_{D_{2 \delta}}\left(u_{0}-\|f\|_{L^{\infty}}\right)_{+} d x\right)^{1-m}+C_{1}(1-m) t\right\}^{\frac{1}{1-m}} \quad \forall t>0
$$

and (2.1) follows.

Proposition 2.2. Let $n \geq 3,0<m<\frac{n-2}{n}, 0<\delta_{1}<\delta_{0}, 0 \leq f \in L^{\infty}(\partial \Omega \times[0, \infty))$ and $0 \leq u_{0} \in L_{l o c}^{p}\left(\bar{\Omega} \backslash\left\{a_{1}, \cdots, a_{i_{0}}\right\}\right)$ for some constant $p>\frac{n(1-m)}{2}$ be such that (1.2) holds for some constants $\lambda_{1}, \cdots, \lambda_{i_{0}} \in \mathbb{R}^{+}$and $\gamma_{1}, \cdots, \gamma_{i_{0}} \in\left(\frac{2}{1-m}, \infty\right)$. Let $u$ be the solution of (1.3) constructed in Theorem 1.1 of HK2]. Then

$$
\left\|u(\cdot, t)-u_{0}\right\|_{L^{1}\left(\Omega_{\delta}\right)} \rightarrow 0 \quad \text { as } t \rightarrow 0 \quad \forall 0<\delta<\delta_{0} .
$$

Proof. Let $0<\delta<\delta_{0}$ and $0<\delta^{\prime}<\delta_{0} / 2$. Then by Lemma2.1,

$$
\begin{aligned}
\left\|u(\cdot, t)-u_{0}\right\|_{L^{1}\left(\Omega_{\delta}\right)} & \leq\left\|u(\cdot, t)-u_{0}\right\|_{L^{1}\left(\Omega_{\delta} \backslash D_{\delta^{\prime}}\right)}+\|u(\cdot, t)\|_{L^{1}\left(D_{\delta^{\prime}}\right)}+\left\|u_{0}\right\|_{L^{1}\left(D_{\delta^{\prime}}\right)} \\
& \leq\left\|u(\cdot, t)-u_{0}\right\|_{L^{1}\left(\Omega_{\delta} \backslash D_{\delta^{\prime}}\right)}+\left(\left\|u_{0}\right\|_{L^{1}\left(D_{2 \delta^{\prime}}\right)}^{1-m}+C t\right)^{\frac{1}{1-m}}+\mid D_{\delta^{\prime}}\|f\|_{L^{\infty}}+\left\|u_{0}\right\|_{L^{1}\left(D_{\delta^{\prime}}\right)} .
\end{aligned}
$$

Letting $t \rightarrow 0$ in (2.4),

$$
\begin{aligned}
& \limsup \left\|u(\cdot, t)-u_{0}\right\|_{L^{1}\left(\Omega_{\delta}\right)} \leq \mid D_{\delta^{\prime}}\|f\|_{L^{\infty}}+2\left\|u_{0}\right\|_{L^{1}\left(D_{2 \delta^{\prime}}\right)} \quad \forall 0<\delta^{\prime}<\delta_{0} / 2 \\
\Rightarrow & \lim _{t \rightarrow 0}\left\|u(\cdot, t)-u_{0}\right\|_{L^{1}\left(\Omega_{\delta}\right)}=0 \quad \text { as } \delta^{\prime} \rightarrow 0
\end{aligned}
$$

and (2.3) follows.

Proof of Theorem 1.1: We will use a modification of the proof of Theorem 6 of [VW2] to prove the theorem. Let $0<\delta<\delta_{0}$ and $t_{1}>t_{0}>0$. Since $u_{1}, u_{2} \in L_{\text {loc }}^{\infty}\left(\bar{\Omega} \backslash\left\{a_{1}, \cdots, i_{0}\right\} \times(0, \infty)\right)$ there exists a constant $M_{1}>0$ such that

$$
u_{j}(x, t) \leq M_{1} \quad \forall x \in \Omega_{\delta}, t_{0} \leq t \leq t_{1}, j=1,2 .
$$

By (1.12) and (2.5), the equation (1.1) for $u_{1}$ and $u_{2}$ are uniformly parabolic on every compact subset of $\bar{\Omega} \backslash\left\{a_{1}, \cdots, i_{0}\right\} \times(0, \infty)$. Hence by the parabolic Schauder estimates [LSU], $u_{1}, u_{2} \in C^{2,1}\left(\bar{\Omega} \backslash\left\{a_{1}, \cdots, i_{0}\right\} \times(0, \infty)\right)$.

We choose a nonnegative monotone increasing function $\phi \in C^{\infty}(\mathbb{R})$ such that $\phi(s)=0$ for any $s \leq 1 / 2$ and $\phi(s)=1$ for any $s \geq 1$. For any $0<\delta<\delta_{0}$, let $\phi_{\delta}(x)=\phi(|x| / \delta)$. Then $\left|\nabla \phi_{\delta}\right| \leq C / \delta$ and $\left|\Delta \phi_{\delta}\right| \leq C / \delta^{2}$. Let $\alpha>\max \left(2+n, \gamma_{1}, \gamma_{2}, \cdots, \gamma_{i_{0}}\right)-n$. We choose $0<\psi \in C^{\infty}\left(\bar{\Omega} \backslash\left\{a_{1}, \cdots, a_{i_{0}}\right\}\right)$ such that $\psi(x)=\left|x-a_{i}\right|^{\alpha}$ for any $x \in \cup_{i=1}^{i_{0}} B_{\delta_{0}}\left(a_{i}\right)$. Let $\delta_{2}=\delta_{1} / 2$ and $T>0$. Then there exists a constant $c_{1}>0$ such that

$$
\psi(x) \geq c_{1} \quad \forall x \in \bar{\Omega} \backslash \cup_{i=1}^{i_{0}} B_{\delta_{2}}\left(a_{i}\right) .
$$


By (1.13) and the choice of $\alpha$, for any $i=1, \cdots, i_{0}$,

$$
\int_{B_{\delta_{2}}\left(a_{i}\right)}\left|x-a_{i}\right|^{\alpha}\left(u_{1}-u_{2}\right)_{+}(x, t) d x \leq C_{T} \int_{0}^{\delta_{2}} \rho^{\alpha+n-\gamma_{i}-1} d \rho=C_{T}^{\prime} \delta_{2}^{\alpha+n-\gamma_{i}}<\infty \quad \forall 0<t<T
$$

for some constants $C_{T}>0, C_{T}^{\prime}>0$. Since $u_{1}, u_{2} \in L_{\text {loc }}^{\infty}\left(\bar{\Omega} \backslash\left\{a_{1}, \cdots, i_{0}\right\} \times(0, \infty)\right)$, by (1.14) and (2.7) for any $T>0$ there exists a constant $C_{0}(T)>0$ such that

$$
\int_{\widehat{\Omega}} \psi(x)\left(u_{1}-u_{2}\right)_{+}(x, t) d x \leq C_{0}(T)<\infty \quad \forall 0<t<T .
$$

Let

$$
w_{\delta}(x)=\prod_{i=1}^{i_{0}} \phi_{\delta}\left(x-a_{i}\right) .
$$

By the Kato inequality $([\overline{\mathrm{DK}}],[\mathrm{K}])$ for any $0<\delta<\delta_{1}, t>0$,

$$
\begin{aligned}
& \frac{\partial}{\partial t}\left(\int_{\widehat{\Omega}}\left(u_{1}-u_{2}\right)_{+} \psi w_{\delta} d x\right) \\
\leq & \int_{\widehat{\Omega}}\left(u_{1}^{m}-u_{2}^{m}\right)_{+} \Delta\left(\psi w_{\delta}\right) d x \\
= & \int_{\widehat{\Omega}}\left\{w_{\delta} \Delta \psi+2 \nabla \psi \cdot \nabla w_{\delta}+\psi \Delta w_{\delta}\right\}\left(u_{1}^{m}-u_{2}^{m}\right)_{+} d x \\
\leq & \int_{\widehat{\Omega}}\left(u_{1}^{m}-u_{2}^{m}\right)_{+} w_{\delta} \Delta \psi d x+\frac{C}{\delta} \sum_{i=1}^{i_{0}} \int_{\delta / 2 \leq\left|x-a_{i}\right| \leq \delta}\left|x-a_{i}\right|^{\alpha-1}\left(u_{1}^{m}-u_{2}^{m}\right)_{+}(x, t) d x \\
& \quad+\frac{C}{\delta^{2}} \sum_{i=1}^{i_{0}} \int_{\delta / 2 \leq\left|x-a_{i}\right| \leq \delta}\left|x-a_{i}\right|^{\alpha}\left(u_{1}^{m}-u_{2}^{m}\right)_{+}(x, t) d x \\
\leq & \int_{\widehat{\Omega}}\left(u_{1}^{m}-u_{2}^{m}\right)_{+} w_{\delta} \Delta \psi d x+C \sum_{i=1}^{i_{0}} \int_{\delta / 2 \leq\left|x-a_{i}\right| \leq \delta}\left|x-a_{i}\right|^{\alpha-2}\left(u_{1}^{m}-u_{2}^{m}\right)_{+}(x, t) d x .
\end{aligned}
$$

By direct computation,

$$
\Delta \psi=\Delta\left|x-a_{i}\right|^{\alpha}=\alpha(\alpha+n-2)\left|x-a_{i}\right|^{\alpha-2} \quad \forall x \in \widehat{B_{\delta_{0}}}\left(a_{i}\right), i=1, \cdots, i_{0} .
$$

By (1.12) and the mean value theorem,

$$
\left(u_{1}^{m}-u_{2}^{m}\right)_{+}(x, t) \leq m \mu_{0}^{m-1}\left(u_{1}-u_{2}\right)_{+}(x, t) \quad \forall x \in \widehat{\Omega}, t>0 .
$$

By (2.6), (2.9), (2.10) and (2.11),

$$
\begin{aligned}
& \frac{\partial}{\partial t}\left(\int_{\widehat{\Omega}}\left(u_{1}-u_{2}\right)_{+} \psi w_{\delta} d x\right) \\
\leq & C \int_{\Omega \mid \cup_{i=1}^{i_{0}} B_{\delta_{2}}\left(a_{i}\right)}\left(u_{1}^{m}-u_{2}^{m}\right)_{+}(x, t) d x+C \int_{\cup_{i=1}^{i_{0}} B_{\delta_{2}}\left(a_{i}\right)}\left|x-a_{i}\right|^{\alpha-2}\left(u_{1}^{m}-u_{2}^{m}\right)_{+}(x, t) d x \\
\leq & C \int_{\widehat{\Omega}}\left(u_{1}-u_{2}\right)_{+}(x, t) \psi(x) d x+C \int_{\cup_{i=1}^{i_{0}} B_{\delta_{2}}\left(a_{i}\right)}\left|x-a_{i}\right|^{\alpha-2}\left(u_{1}^{m}-u_{2}^{m}\right)_{+}(x, t) d x .
\end{aligned}
$$


By (1.13) and the mean value theorem for any $\left|x-a_{i}\right| \leq \delta_{2}, 0<t<T, i=1, \cdots, i_{0}$,

$$
\begin{aligned}
\left|x-a_{i}\right|^{\alpha-2}\left(u_{1}^{m}-u_{2}^{m}\right)_{+}(x, t) & \leq m\left|x-a_{i}\right|^{\alpha-2} u_{2}(x, t)^{m-1}\left(u_{1}-u_{2}\right)_{+}(x, t) \\
& \leq m C_{1}(T)^{m-1}\left|x-a_{i}\right|^{(1-m) \gamma_{i}-2+\alpha}\left(u_{1}-u_{2}\right)_{+}(x, t) \\
& \leq m C_{1}(T)^{m-1} \delta_{0}^{(1-m) \gamma_{i}-2}\left|x-a_{i}\right|^{\alpha}\left(u_{1}-u_{2}\right)_{+}(x, t) \\
& \leq m C_{1}(T)^{m-1} \delta_{0}^{(1-m) \gamma_{i}-2} \psi(x)\left(u_{1}-u_{2}\right)_{+}(x, t) .
\end{aligned}
$$

By (2.12) and (2.13),

$$
\frac{\partial}{\partial t}\left(\int_{\widehat{\Omega}}\left(u_{1}-u_{2}\right)_{+} \psi w_{\delta} d x\right) \leq C_{T} \int_{\widehat{\Omega}}\left(u_{1}-u_{2}\right)_{+}(x, t) \psi(x) d x
$$

Integrating (2.14) over $(0, t)$, by (1.14) and (2.8),

$$
\begin{aligned}
& \int_{\widehat{\Omega}}\left(u_{1}-u_{2}\right)_{+}(x, t) \psi(x) w_{\delta}(x) d x \leq C_{T} \int_{0}^{t} \int_{\widehat{\Omega}}\left(u_{1}-u_{2}\right)_{+}(x, t) \psi(x) d x d t \quad \forall 0<t<T \\
\Rightarrow & \int_{\widehat{\Omega}}\left(u_{1}-u_{2}\right)_{+}(x, t) \psi(x) d x \leq C_{T} \int_{0}^{t} \int_{\widehat{\Omega}}\left(u_{1}-u_{2}\right)_{+}(x, t) \psi(x) d x d t \quad \forall 0<t<T \quad \text { as } \delta \rightarrow 0 .
\end{aligned}
$$

By (2.15),

$$
u_{1}(x, t) \leq u_{2}(x, t) \quad \forall x \in \widehat{\Omega}, 0<t<T .
$$

Letting $T \rightarrow \infty$ in (2.16) we get (1.15) and the theorem follows.

By Theorem 1.1, Lemma 2.3, Lemma 2.15 of [HK2] and Theorem 1.1 and Proposition 2.2 we have the following result.

Theorem 2.3. Let $n \geq 3,0<m<\frac{n-2}{n}, 0<\delta_{1}<\min \left(1, \delta_{0}\right), \mu_{0}>0, f \in C^{3}(\partial \Omega \times(0, \infty)) \cap$ $L^{\infty}(\partial \Omega \times(0, \infty))$ be such that $f \geq \mu_{0}$ on $\partial \Omega \times(0, \infty)$ and $\mu_{0} \leq u_{0} \in L_{\text {loc }}^{p}\left(\bar{\Omega} \backslash\left\{a_{1}, \cdots, a_{i_{0}}\right\}\right)$ for some constant $p>\frac{n(1-m)}{2}$ be such that (1.2) and (1.6) hold for some constants satisfying (1.11) and $\lambda_{1}$, $\cdots, \lambda_{i_{0}}, \lambda_{1}^{\prime}, \cdots, \lambda_{i_{0}}^{\prime} \in \mathbb{R}^{+}$. Then there exists a unique solution $u$ of (1.3) which satisfies (1.8) and (2.3) such that for any constants $T>0$ and $\delta_{2} \in\left(0, \delta_{1}\right)$ there exist constants $C_{1}=C_{1}(T)>0$, $C_{2}=C_{2}(T)>0$, depending only on $\lambda_{1}, \cdots, \lambda_{i_{0}}, \lambda_{1}^{\prime}, \cdots, \lambda_{i_{0}}^{\prime}, \gamma_{1}, \cdots, \gamma_{i_{0}}, \gamma_{1^{\prime}}^{\prime}, \cdots, \gamma_{i_{0}}^{\prime}$, such that

$$
\frac{C_{1}}{\left|x-a_{i}\right|^{\gamma_{i}}} \leq u(x, t) \leq \frac{C_{2}}{\left|x-a_{i}\right|^{\gamma_{i}^{\prime}}} \quad \forall 0<\left|x-a_{i}\right|<\delta_{2}, 0<t<T, i=1,2, \ldots, i_{0}
$$

holds.

Proof of Theorem 1.2: We will use a modification of the proof of Lemma 2.3 of [DaK] to prove the theorem. Let

$$
A=A(x, t)= \begin{cases}\frac{u_{1}(x, t)^{m}-u_{2}(x, t)^{m}}{u_{1}(x, t)-u_{2}(x, t)} & \forall x \in \widehat{\Omega}, t>0 \text { satisfying } u_{1}(x, t) \neq u_{2}(x, t) \\ m u_{1}(x, t)^{m-1} & \forall x \in \widehat{\Omega}, t>0 \text { satisfying } u_{1}(x, t)=u_{2}(x, t) \\ 0 & \forall x=a_{i}, i=1, \cdots, i_{0}, t>0 .\end{cases}
$$


For any $k \in \mathbb{Z}^{+}$, let

$$
\alpha_{k}(x, t)=\left\{\begin{array}{lr}
\frac{\left|u_{1}(x, t)^{m}-u_{2}(x, t)^{m}\right|}{\left|u_{1}(x, t)-u_{2}(x, t)\right|+(1 / k)} \quad \forall x \in \widehat{\Omega}, t>0 \\
0 \quad \forall x=a_{i}, i=1, \cdots, i_{0}, t>0
\end{array}\right.
$$

and $A_{k}=A_{k}(x, t)=\alpha_{k}(x, t)+k^{-1}$. We choose a nonnegative monotone increasing function $\phi \in C^{\infty}(\mathbb{R})$ such that $\phi(s)=0$ for any $s \leq 0$ and $\phi(s)=1$ for any $s \geq 1$. Let $0<\delta_{2} \leq \delta_{1} / 2$. For any $\delta \in\left(0, \delta_{2} / 2\right)$ and $j \geq 2 / \delta_{2}$, let $\phi_{j}(x)=\phi(j(|x|-\delta))$. Let $t_{1}>t_{0}>0$ and $0 \leq h \in C_{0}^{\infty}\left(\Omega_{\delta_{2}}\right)$. For any $k \in \mathbb{Z}^{+}$and $0<\delta \leq \delta_{2} / 2$, let $\psi_{k, \delta}$ be the solution of

$$
\begin{cases}\psi_{t}+A_{k} \Delta \psi=0 & \text { in } \Omega_{\delta} \times\left(0, t_{1}\right) \\ \psi(x, t)=0 & \text { on } \partial \Omega_{\delta} \times\left(0, t_{1}\right) \\ \psi\left(x, t_{0}\right)=h(x) & \text { in } \Omega_{\delta}\end{cases}
$$

and

$$
w_{j}(x)=\prod_{i=1}^{i_{0}} \phi_{j}\left(x-a_{i}\right) .
$$

Then $\left|\nabla w_{j}\right| \leq C j$ and $\left|\Delta w_{j}\right| \leq C j^{2}$ for some constant $C>0$. By the maximum principle, $0 \leq \psi_{k, \delta} \leq\|h\|_{L^{\infty}}$ in $\Omega_{\delta} \times\left(0, t_{1}\right)$. Hence $\frac{\partial \psi_{k, \delta}}{\partial v} \leq 0$ on $\partial \Omega \times\left(0, t_{1}\right)$. Then

$$
\begin{aligned}
& \quad \int_{\Omega_{\delta}}\left(u_{1}\left(x, t_{1}\right)-u_{2}\left(x, t_{1}\right)\right) h(x) d x \\
& =\int_{\Omega_{\delta}}\left(u_{1}\left(x, t_{0}\right)-u_{2}\left(x, t_{0}\right)\right) \psi_{k, \delta}\left(x, t_{0}\right) w_{j}(x) d x+\int_{t_{0}}^{t_{1}} \int_{\partial \Omega}\left(f_{2}^{m}-f_{1}^{m}\right) \frac{\partial \psi_{k, \delta}}{\partial v} d \sigma d t \\
& \quad+\int_{t_{0}}^{t_{1}} \int_{\Omega_{\delta}}\left(u_{1}-u_{2}\right)\left\{w_{j}\left(\partial_{t} \psi_{k, \delta}+A \Delta \psi_{k, \delta}\right)+A \nabla w_{j} \cdot \nabla \psi_{k, \delta}+A \psi_{k, \delta} \Delta w_{j}\right\} d x d t \\
& \quad \leq|| h\left\|_{L^{\infty}} \int_{\Omega_{\delta}}\left(u_{1}\left(x, t_{0}\right)-u_{2}\left(x, t_{0}\right)\right)_{+} d x+\int_{t_{0}}^{t_{1}} \int_{\Omega_{\delta}}\left|u_{1}-u_{2}\right|\left|A-A_{k} \| \Delta \psi_{k, \delta}\right| d x d t\right. \\
& \quad+C \sum_{i=1}^{i_{0}} \int_{t_{0}}^{t_{1}} \int_{\delta \leq\left|x-a_{i}\right| \leq \delta+j^{-1}}\left|u_{1}^{m}-u_{2}^{m}\right|\left\{j|\nabla| x-a_{i}\left|\cdot \nabla \psi_{k, \delta}\right|+j^{2} \psi_{k, \delta}\right\} d x d t \\
& =I_{1}+I_{2}+I_{3} .
\end{aligned}
$$

We will now use a modification of the proof of Theorem 2.1 of [PV] to estimate the derivative of $\psi_{k, \delta}$ on $\cup_{i=1}^{i_{0}} \partial B_{\delta}\left(a_{i}\right) \times\left(0, t_{1}\right)$. Let

$$
q_{i}(x)=\frac{\delta^{2-n}-\left|x-a_{i}\right|^{2-n}}{\delta^{2-n}-\delta_{2}^{2-n}} \cdot\|h\|_{L^{\infty}} \quad \forall i=1, \cdots, i_{0} .
$$

Then for any $i=1, \cdots, i_{0}, q_{i}$ satisfies

$$
\left\{\begin{aligned}
q_{t}+A_{k} \Delta q & =0 & & \text { in }\left(B_{\delta_{2}}\left(a_{i}\right) \backslash \overline{B_{\delta}\left(a_{i}\right)}\right) \times\left(0, t_{1}\right) \\
q & =0 & & \text { on } \partial B_{\delta}\left(a_{i}\right) \times\left(0, t_{1}\right) \\
q & =\|h\|_{L^{\infty}} & & \text { on } \partial B_{\delta_{2}}\left(a_{i}\right) \times\left(0, t_{1}\right) \\
q & \geq 0 & & \text { on } B_{\delta_{2}}\left(a_{i}\right) \backslash \overline{B_{\delta}\left(a_{i}\right)}
\end{aligned}\right.
$$


Since $\psi_{k, \delta}$ is a subsolution of (2.21), by the maximum principle,

$$
\begin{aligned}
& 0 \leq \psi_{k, \delta}(x, t) \leq q_{i}(x) \quad \forall \delta \leq\left|x-a_{i}\right| \leq \delta_{2}, 0<t \leq t_{1}, i=1, \cdots, i_{0} \\
\Rightarrow & \left|\frac{\partial \psi_{k, \delta}}{\partial v}\right| \leq\left|\frac{\partial q_{i}}{\partial v}\right|=\frac{(n-2) \delta^{1-n}}{\delta^{2-n}-\delta_{2}^{2-n}}\|h\|_{L^{\infty}} \quad \text { on } \partial B_{\delta}\left(a_{i}\right) \times\left(0, t_{1}\right) \quad \forall i=1, \cdots, i_{0} .
\end{aligned}
$$

By (2.20) and the mean value theorem,

$$
q_{i}(x) \leq \frac{(n-2) j^{-1} \delta^{1-n}}{\delta^{2-n}-\delta_{2}^{2-n}}\|h\|_{L^{\infty}} \quad \forall \delta \leq\left|x-a_{i}\right| \leq \delta+j^{-1}, i=1, \cdots, i_{0} .
$$

By (1.13), (1.16), (2.19), (2.22), (2.23) and (2.24),

$$
\begin{aligned}
I_{3} & \leq C \sum_{i=1}^{i_{0}} j \int_{t_{0}}^{t_{1}} \int_{\delta \leq\left|x-a_{i}\right| \leq \delta+j^{-1}}\left|u_{1}^{m}-u_{2}^{m}\right|\left\{|\nabla| x-a_{i}\left|\cdot \nabla \psi_{k, \delta}\right|+\frac{(n-2) \delta^{1-n}}{\delta^{2-n}-\delta_{2}^{2-n}}\|h\|_{L^{\infty}}\right\} d x d t \\
& =C \sum_{i=1}^{i_{0}} \int_{t_{0}}^{t_{1}} \int_{\partial B_{\delta}\left(a_{i}\right)}\left|u_{1}^{m}-u_{2}^{m}\right|\left\{\left|\frac{\partial \psi_{k, \delta}}{\partial v}\right|+\frac{(n-2) \delta^{1-n}}{\delta^{2-n}-\delta_{2}^{2-n}}\|h\|_{L^{\infty}}\right\} d x d t \quad \text { as } j \rightarrow \infty \\
& \leq 2 C \frac{(n-2) \delta^{1-n}}{\delta^{2-n}-\delta_{2}^{2-n}}\|h\|_{L^{\infty}} \sum_{i=1}^{i_{0}} \int_{t_{0}}^{t_{1}} \int_{\partial B_{\delta}\left(a_{i}\right)}\left|u_{1}^{m}-u_{2}^{m}\right| d \sigma d t \\
& \leq \frac{C^{\prime}|| h \|_{L^{\infty}} t_{1} \delta^{1-n}}{\delta^{2-n}-\delta_{2}^{2-n}} \sum_{i=1}^{i_{0}} \delta^{n-1-m \gamma_{i}^{\prime}} .
\end{aligned}
$$

By the same argument as the proof of Lemma 2.3 of [DK],

$$
\lim _{k \rightarrow \infty} I_{2}=0 \text {. }
$$

Hence letting first $j \rightarrow \infty$ and then $k \rightarrow \infty$ in (2.19), by (2.25) and (2.26),

$$
\begin{aligned}
& \int_{\Omega_{\delta}}\left(u_{1}\left(x, t_{1}\right)-u_{2}\left(x, t_{1}\right)\right) h(x) d x \\
\leq & \|h\|_{L^{\infty}} \int_{\Omega_{\delta}}\left(u_{1}\left(x, t_{0}\right)-u_{2}\left(x, t_{0}\right)\right)_{+} d x+\frac{C^{\prime}\|h\|_{L^{\infty}} t_{1} \delta^{1-n}}{\delta^{2-n}-\delta_{2}^{2-n}} \sum_{i=1}^{i_{0}} \delta^{n-1-m \gamma_{i}^{\prime}} .
\end{aligned}
$$

Letting $t_{0} \rightarrow 0$ in (2.27), by (1.14) and (1.16),

$$
\begin{aligned}
& \int_{\Omega_{\delta}}\left(u_{1}\left(x, t_{1}\right)-u_{2}\left(x, t_{1}\right)\right) h(x) d x \leq \frac{C^{\prime}\|h\|_{L^{\infty}} t_{1} \delta^{2-n}}{\delta^{2-n}-\delta_{2}^{2-n}} \sum_{i=1}^{i_{0}} \delta^{n-2-m \gamma_{i}^{\prime}} \\
\Rightarrow & \int_{\widehat{\Omega}}\left(u_{1}\left(x, t_{1}\right)-u_{2}\left(x, t_{1}\right)\right) h(x) d x=0 \quad \forall t_{1}>0 \quad \text { as } \delta \rightarrow 0 .
\end{aligned}
$$


We now choose a sequence of smooth functions $0 \leq h_{i} \in C_{0}^{\infty}\left(\Omega_{\delta_{2}}\right)$ such that $h_{i}(x) \rightarrow$ $\chi_{\left\{u_{1}>u_{2}\right\} \cap \Omega_{\delta_{2}}}(x)$ for any $x \in \Omega_{\delta_{2}}$ as $i \rightarrow \infty$. Putting $h=h_{i}$ in (2.28) and letting $i \rightarrow \infty$,

$$
\begin{aligned}
& \int_{\Omega_{\delta_{2}}}\left(u_{1}\left(x, t_{1}\right)-u_{2}\left(x, t_{1}\right)\right)_{+} d x=0 \quad \forall t_{1}>0,0<\delta_{2}<\delta_{1} / 2 \\
\Rightarrow & \int_{\widehat{\Omega}}\left(u_{1}\left(x, t_{1}\right)-u_{2}\left(x, t_{1}\right)\right)_{+} d x=0 \quad \forall t_{1}>0 \quad \text { as } \delta_{2} \rightarrow 0
\end{aligned}
$$

and (1.15) follows.

By Theorem 1.1, Lemma 2.3, Lemma 2.15 of [HK2] and Theorem 1.2 and Proposition 2.2 we have the following result.

Theorem 2.4. Let $n \geq 3,0<m<\frac{n-2}{n}, 0<\delta_{1}<\min \left(1, \delta_{0}\right), \mu_{0}>0, f \in L^{\infty}(\partial \Omega \times(0, \infty))$ be such that $f \geq \mu_{0}$ on $\partial \Omega \times(0, \infty)$ and $\mu_{0} \leq u_{0} \in L_{\text {loc }}^{p}\left(\bar{\Omega} \backslash\left\{a_{1}, \cdots, a_{i_{0}}\right\}\right)$ for some constant $p>\frac{n(1-m)}{2}$ be such that (1.2) and (1.6) hold for some constants satisfying (1.16) and $\lambda_{1}, \cdots, \lambda_{i_{0}}, \lambda_{1}^{\prime}, \cdots$, $\lambda_{i_{0}}^{\prime} \in \mathbb{R}^{+}$. Then there exists a unique solution $u$ of (1.3) which satisfies (2.3) such that for any constants $T>0$ and $\delta_{2} \in\left(0, \delta_{1}\right)$ there exist constants $C_{1}=C_{1}(T)>0, C_{2}=C_{2}(T)>0$, such that (2.17) holds.

Proof of Theorem 1.3. Since the proof is similar to the proof of Theorem 1.2, we will only sketch the argument here. Let

$$
A=A(x, t)= \begin{cases}\frac{u_{1}(x, t)^{m}-u_{2}(x, t)^{m}}{u_{1}(x, t)-u_{2}(x, t)} & \forall x \in \widehat{\mathbb{R}^{n}}, t>0 \text { satisfying } u_{1}(x, t) \neq u_{2}(x, t) \\ m u_{1}(x, t)^{m-1} & \forall x \in \widehat{\mathbb{R}^{n}}, t>0 \text { satisfying } u_{1}(x, t)=u_{2}(x, t) \\ 0 & \forall x=a_{i}, i=1, \cdots, i_{0}, t>0 .\end{cases}
$$

For any $k \in \mathbb{Z}^{+}$, let

$$
\alpha_{k}(x, t)= \begin{cases}\frac{\left|u_{1}(x, t)^{m}-u_{2}(x, t)^{m}\right|}{\left|u_{1}(x, t)-u_{2}(x, t)\right|+(1 / k)} & \forall x \in \widehat{\mathbb{R}^{n}}, t>0 \\ 0 & \forall x=a_{i}, i=1, \cdots, i_{0}, t>0\end{cases}
$$

and $A_{k}=A_{k}(x, t)=\alpha_{k}(x, t)+k^{-1}$. Let $0<\delta_{2} \leq \delta_{1} / 2$. For any $\delta \in\left(0, \delta_{2} / 2\right)$ and $j \geq 2 / \delta_{2}$, let $\phi, \phi_{j}$ and $w_{j}$ be as in the proof of Theorem 1.2. Let $t_{1}>t_{0}>0, R_{0}^{\prime}>R_{1}+1, R>2 R_{0}^{\prime}$ and $h \in C_{0}^{\infty}\left(\Omega_{\delta_{2}, R_{0}^{\prime}}\right)$. For any $k \in \mathbb{Z}^{+}$and $0<\delta \leq \delta_{2} / 2$, let $\psi_{k, \delta, R}$ be the solution of

$$
\begin{cases}\psi_{t}+A_{k} \Delta \psi=0 & \text { in } \Omega_{\delta, R} \times\left(0, t_{1}\right) \\ \psi(x, t)=0 & \text { on } \partial \Omega_{\delta, R} \times\left(0, t_{1}\right) \\ \psi\left(x, t_{0}\right)=h(x) & \text { in } \Omega_{\delta, R}\end{cases}
$$


By the maximum principle, $0 \leq \psi_{k, \delta, R} \leq\|h\|_{L^{\infty}}$ in $\Omega_{\delta, R} \times\left(0, t_{1}\right)$. Hence $\frac{\partial \psi_{k, \delta, R}}{\partial v} \leq 0$ on $\partial B_{R} \times\left(0, t_{1}\right)$. Then by an argument similar to the proof of Theorem 1.2 ,

$$
\begin{aligned}
& \int_{\Omega_{\delta, R}}\left(u_{1}\left(x, t_{1}\right)-u_{2}\left(x, t_{1}\right)\right) h(x) d x \\
\leq\|h\|_{L^{\infty}} & \int_{\Omega_{\delta, R}}\left(u_{1}\left(x, t_{0}\right)-u_{2}\left(x, t_{0}\right)\right)_{+} d x+\int_{t_{0}}^{t_{1}} \int_{\Omega_{\delta, R}}\left|u_{1}-u_{2}\left\|A-A_{k}\right\| \Delta \psi_{k, \delta, R}\right| d x d t \\
& +\int_{t_{0}}^{t_{1}} \int_{\partial B_{R}}\left|u_{1}(x, t)^{m}-u_{2}(x, t)^{m}\right|\left|\frac{\partial \psi_{k, \delta, R}}{\partial v}\right| d \sigma d t \\
& +\frac{C \delta^{2-n}}{\delta^{2-n}-\delta_{2}^{2-n}}\|h\|_{L^{\infty}} \sum_{i=1}^{i_{0}} \delta^{n-2-m \gamma_{i}^{\prime}} .
\end{aligned}
$$

Let

$$
Q(x)=\frac{|x|^{2-n}-R^{2-n}}{(R / 2)^{2-n}-R^{2-n}}\|h\|_{L^{\infty}} .
$$

Then $Q$ satisfies

$$
\left\{\begin{aligned}
q_{t}+A_{k} \Delta q & =0 & & \text { in }\left(B_{R} \backslash \overline{B_{R / 2}}\right) \times\left(0, t_{1}\right) \\
q & =0 & & \text { on } \partial B_{R} \times\left(0, t_{1}\right) \\
q & =\|h\|_{L^{\infty}} & & \text { on } \partial B_{R / 2} \times\left(0, t_{1}\right) \\
q & \geq 0 & & \text { on } B_{R} \backslash B_{R / 2}
\end{aligned}\right.
$$

Since $\psi_{k, \delta, R}$ is a subsolution of (2.33), by the maximum principle,

$$
\begin{aligned}
& 0 \leq \psi_{k, \delta, R}(x, t) \leq Q(x) \quad \forall R / 2 \leq|x| \leq R, 0<t \leq t_{1} \\
\Rightarrow & \left|\frac{\partial \psi_{k, \delta, R}}{\partial v}\right| \leq\left|\frac{\partial Q}{\partial v}\right|=\frac{(n-2) R^{1-n}}{(R / 2)^{2-n}-R^{2-n}}\|h\|_{L^{\infty}} \leq \frac{C}{R}\|h\|_{L^{\infty}} \quad \text { on } \partial B_{R} \times\left(0, t_{1}\right) .
\end{aligned}
$$

By (2.32) and (2.34),

$$
\begin{aligned}
& \int_{\Omega_{\delta, R}}\left(u_{1}\left(x, t_{1}\right)-u_{2}\left(x, t_{1}\right)\right) h(x) d x \\
& \leq\|h\|_{L^{\infty}} \int_{\Omega_{\delta, R}}\left(u_{1}\left(x, t_{0}\right)-u_{2}\left(x, t_{0}\right)\right)_{+} d x+\int_{t_{0}}^{t_{1}} \int_{\Omega_{\delta, R}}\left|u_{1}-u_{2}\left\|A-A_{k}\right\| \Delta \psi_{k, \delta, R}\right| d x d t \\
& \quad+\frac{C\|h\|_{L^{\infty}}}{R} \int_{t_{0}}^{t_{1}} \int_{\partial B_{R}}\left|u_{1}(x, t)^{m}-u_{2}(x, t)^{m}\right| d \sigma d t \\
& \quad+\frac{C \delta^{2-n}}{\delta^{2-n}-\delta_{2}^{2-n}}\|h\|_{L^{\infty}} \sum_{i=1}^{i_{0}} \delta^{n-2-m \gamma_{i}^{\prime}} .
\end{aligned}
$$

Letting first $k \rightarrow \infty$ and then $t_{0} \rightarrow 0, \delta \rightarrow 0$ in (2.35), by the proof of Lemma 2.3 of [DK] and similar argument as the proof of Theorem 1.2, the first term, second term and the last 
term on the right hand side of (2.35) vanish. This together with the mean value theorem and (1.19) implies that

$$
\begin{aligned}
& \int_{\widehat{B}_{R}}\left(u_{1}\left(x, t_{1}\right)-u_{2}\left(x, t_{1}\right)\right) h(x) d x \\
\leq & \frac{C\|h\|_{L^{\infty}}}{R} \int_{t_{0}}^{t_{1}} \int_{\partial B_{R}}\left|u_{1}(x, t)^{m}-u_{2}(x, t)^{m}\right| d \sigma d t \\
\leq & \frac{m C \mu_{0}^{m-1}\|h\|_{L^{\infty}}}{R} \int_{0}^{t_{1}} \int_{\partial B_{R}}\left|u_{1}(x, t)-u_{2}(x, t)\right| d \sigma d t \\
\leq & \frac{C^{\prime}\|h\|_{L^{\infty}}}{R}\left\{\int_{0}^{t_{1}} \int_{\partial B_{R}}\left|u_{1}(x, t)-\mu_{0}\right| d \sigma d t+\int_{0}^{t_{1}} \int_{\partial B_{R}}\left|u_{1}(x, t)-\mu_{0}\right| d \sigma d t\right\} .
\end{aligned}
$$

By (1.20) there exists a sequence $\left\{R_{j}\right\}_{j=2}^{\infty} \subset\left(2 R_{0}^{\prime}, \infty\right), R_{j} \rightarrow \infty$ as $j \rightarrow \infty$, such that

$$
\int_{0}^{t_{1}} \int_{\partial B_{R_{j}}}\left(\left|u_{1}(x, t)-\mu_{0}\right|+\left|u_{2}(x, t)-\mu_{0}\right|\right) d \sigma d t \rightarrow 0 \quad \text { as } j \rightarrow \infty .
$$

Putting $R=R_{j}$ in (2.36) and letting $j \rightarrow \infty$, by (2.37),

$$
\int_{\widehat{\mathbb{R}^{n}}}\left(u_{1}\left(x, t_{1}\right)-u_{2}\left(x, t_{1}\right)\right) h(x) d x=0 \quad \forall t_{1}>0
$$

By (2.38) and an argument similar to the proof of Theorem 1.2,

$$
\int_{\widehat{\mathbb{R}}^{n}}\left(u_{1}\left(x, t_{1}\right)-u_{2}\left(x, t_{1}\right)\right)_{+} d x=0 \quad \forall t_{1}>0
$$

and (1.21) follows.

By Theorem 1.2, Lemma 2.3, Lemma 2.15 and the proof of Theorem 1.6 of [HK2] and Theorem 1.3 we have the following result.

Theorem 2.5. Let $n \geq 3,0<m<\frac{n-2}{n}, 0<\delta_{1}<\min \left(1, \delta_{0}\right), \mu_{0}>0$ and $\mu_{0} \leq u_{0} \in$ $L_{\text {loc }}^{p}\left(\widehat{\mathbb{R}^{n}} \backslash\left\{a_{1}, \cdots, a_{i_{0}}\right\}\right)$ for some constant $p>\frac{n(1-m)}{2}$ be such that (1.10) holds for some constants satisfying (1.18) and $\lambda_{1}, \cdots, \lambda_{i_{0}}, \lambda_{1}^{\prime}, \cdots, \lambda_{i_{0}}^{\prime} \in \mathbb{R}^{+}$. Suppose (1.17) also holds for some constant $R_{1}>R_{0}$. Then there exists a unique solution $u$ of (1.4) which satisfy

$$
u(x, t) \geq \mu_{0} \quad \forall x \in \widehat{\mathbb{R}^{n}}, t>0
$$

and

$$
\int_{\widehat{\mathbb{R}^{n}}}\left|u(x, t)-\mu_{0}\right| d x \leq \int_{\widehat{\mathbb{R}^{n}}}\left|u_{0}-\mu_{0}\right| d x \quad \forall t>0
$$

such that for any constants $T>0$ and $\delta_{2} \in\left(0, \delta_{1}\right)$ there exist constants $C_{1}=C_{1}(T)>0$, $C_{2}=C_{2}(T)>0$, such that (2.17) holds. 


\section{Existence of highly oscillating solution}

In this section we will prove the existence of initial data such that the corresponding solution of (1.23) oscillates between infinity and some positive constant as $t \rightarrow \infty$. We start with a stability result for the solutions of (1.23).

Lemma 3.1. Let $n \geq 3,0<m<\frac{n-2}{n}, 0<\delta_{1}<\min \left(1, \delta_{0}\right), \mu_{0}>0$. Let $\left\{u_{0, j}\right\}_{j=1}^{\infty} \subset L_{l o c}^{p}(\bar{\Omega} \backslash$ $\left.\left\{a_{1}, \cdots, a_{i_{0}}\right\}\right)$ for some constant $p>\frac{n(1-m)}{2}$ be a sequence of functions satisfying

$$
u_{0, j} \geq \mu_{0} \quad \text { on } \bar{\Omega} \backslash\left\{a_{1}, \cdots, a_{i_{0}}\right\} \quad \forall j \in \mathbb{Z}^{+}
$$

such that

$$
\frac{\lambda_{i}}{\left|x-a_{i}\right|^{\gamma_{i}}} \leq u_{0, j}(x) \leq \frac{\lambda_{i}^{\prime}}{\left|x-a_{i}\right|^{\gamma_{i}^{\prime}}} \quad \forall 0<\left|x-a_{i}\right|<\delta_{1}, i=1, \cdots, i_{0}, j \in \mathbb{Z}^{+}
$$

holds for some constants satisfying (1.11), $\lambda_{1}, \cdots, \lambda_{i_{0}}, \lambda_{1^{\prime}}^{\prime} \cdots, \lambda_{i_{0}}^{\prime} \in \mathbb{R}^{+}$. Let $\mu_{0} \leq u_{0} \in$ $L_{\text {loc }}^{p}\left(\bar{\Omega} \backslash\left\{a_{1}, \cdots, a_{i_{0}}\right\}\right)$ be such that (1.22) holds. Let $u, u_{j}, j \in \mathbb{Z}^{+}$, be the unique solutions of (1.23) with initial value $u_{0}, u_{0, j}$ respectively, given by Theorem 2.3. Suppose

$$
u_{0, j} \rightarrow u_{0} \quad \text { in } L_{l o c}^{p}\left(\bar{\Omega} \backslash\left\{a_{1}, \cdots, a_{i_{0}}\right\}\right) \quad \text { as } j \rightarrow \infty .
$$

Then $u_{j}$ converges to $u$ uniformly in $C^{2,1}\left(\Omega_{\delta} \times\left(t_{1}, t_{2}\right)\right)$ as $j \rightarrow \infty$ for any $0<\delta<\delta_{0}$ and $t_{2}>t_{1}>0$. Proof. Let $0<\delta^{\prime}<\delta<\delta_{0}$ and $t_{2}>t_{1}>0$. By (3.3) there exists a constant $M_{1}>0$ such that

$$
\left\|u_{0, j}\right\|_{L^{p}\left(\Omega_{\delta^{\prime}}\right)} \leq M_{1} \quad \forall j \in \mathbb{Z}^{+} .
$$

By (3.4) and Lemma 2.9 of [HK2] there exists a constant $M_{2}>0$ depending on $M_{1}$ and $\mu_{0}$ such that

$$
\left\|u_{j}\right\|_{L^{\infty}\left(\Omega_{\delta} \times\left(t_{1} / 2, t_{2}\right)\right)} \leq M_{2} \quad \forall j \in \mathbb{Z}^{+} .
$$

By Theorem 2.3.

$$
u_{j} \geq \mu_{0} \quad \text { in } \bar{\Omega} \backslash\left\{a_{1}, \cdots, a_{i_{0}}\right\} \times(0, \infty) \quad \forall j \in \mathbb{Z}^{+} .
$$

By (3.5) and (3.6) the equation (1.1) for $u_{j}$ are uniformly parabolic on every compact subset of $\bar{\Omega} \backslash\left\{a_{1}, \cdots, a_{i_{0}}\right\} \times(0, \infty)$. Hence by the Ascoli theorem, diagonalization argument, and an argument similar to the proof of Lemma 2.11 of [HK2] and Theorem 1.1 of [HK1] the sequence $\left\{u_{j}\right\}_{j=1}^{\infty}$ has a subsequence $\left\{u_{j_{k}}\right\}_{k=1}^{\infty}$ that converges uniformly in $C^{2,1}\left(\Omega_{\delta} \times\left(t_{1}, t_{2}\right)\right)$ to a solution $v$ of (1.23) as $k \rightarrow \infty$ for any $0<\delta<\delta_{0}$ and $t_{2}>t_{1}>0$ and

$$
v \geq \mu_{0} \quad \text { in } \widehat{\Omega} \times(0, \infty) .
$$

Since by Theorem 2.3 for any $T>0$ there exists constants $C_{1}=C_{1}(T)>0, C_{2}=C_{2}(T)>0$, such that (2.17) holds for any $u_{j}$, putting $u=u_{j_{k}}$ in (2.17) and letting $k \rightarrow \infty$,

$$
\frac{C_{1}}{\left|x-a_{i}\right|^{\gamma_{i}}} \leq v(x, t) \leq \frac{C_{2}}{\left|x-a_{i}\right|_{i}^{\prime}} \quad \forall 0<\left|x-a_{i}\right|<\delta_{2}, 0<t<T, i=1,2, \ldots, i_{0} .
$$


By Lemma 2.1 there exists a constant $C>0$ such that

$$
\begin{aligned}
\int_{D_{\delta_{1}}} u_{j}(x, t) d x & \leq\left\{\left(\int_{D_{2 \delta_{1}}} u_{0, j} d x\right)^{1-m}+C t\right\}^{\frac{1}{1-m}}+\left|D_{\delta_{1}}\right| \mu_{0} \quad \forall t>0,0<\delta_{1}<\delta_{0} / 2, j \in \mathbb{Z}^{+} \\
& \left.\leq\left\{\left(\left|D_{2 \delta_{1}}\right|^{1-\frac{1}{p}}\left\|u_{0, j}\right\|_{L^{p}\left(D_{2 \delta_{1}}\right)}\right)\right)^{1-m}+C t\right\}^{\frac{1}{1-m}}+\left|D_{\delta_{1}}\right| \mu_{0} \quad \forall t>0,0<\delta_{1}<\delta_{0} / 2, j \in \mathbb{Z}^{+} .
\end{aligned}
$$

Let $\varepsilon>0$. By (3.3) there exists $j_{0} \in \mathbb{Z}^{+}$such that

$$
\left\|u_{0, j}\right\|_{L^{p}\left(D_{\delta_{1}}\right)} \leq\left\|u_{0}\right\|_{L^{p}\left(D_{\delta_{1}}\right)}+\varepsilon \quad \forall 0<\delta_{1}<\delta_{0}, j \geq j_{0} .
$$

By (3.9), (3.10) and Holder's inequality,

$$
\begin{aligned}
& \int_{\Omega_{\delta}}\left|u_{j}(x, t)-u_{0, j}(x)\right| d x \\
& \leq \int_{\Omega_{\delta} \backslash D_{\delta_{1}}}\left|u_{j}(x, t)-u_{0, j}(x)\right| d x+\left\{\left(\left|D_{2 \delta_{1}}\right|^{1-\frac{1}{p}}\left(\left\|u_{0}\right\|_{L^{p}\left(D_{2 \delta_{1}}\right)}+\varepsilon\right)\right)^{1-m}+C t\right\}^{\frac{1}{1-m}}+\left|D_{\delta_{1}}\right| \mu_{0} \\
& \quad+\left|D_{\delta_{1}}\right|^{1-\frac{1}{p}}\left(\left\|u_{0}\right\|_{L^{p}\left(D_{\delta_{1}}\right)}+\varepsilon\right) \quad \forall 0<\delta_{1}<\delta_{0} / 2, t>0, j \geq j_{0} .
\end{aligned}
$$

Leting $j=j_{k} \rightarrow \infty$ in (3.11),

$$
\begin{aligned}
& \int_{\Omega_{\delta}}\left|v(x, t)-u_{0}(x)\right| d x \\
& \leq \int_{\Omega_{\delta} \backslash D_{\delta_{1}}}\left|v(x, t)-u_{0}(x)\right| d x+\left\{\left(\left|D_{2 \delta_{1}}\right|^{1-\frac{1}{p}}\left(\left\|u_{0}\right\|_{L^{p}\left(D_{2 \delta_{1}}\right)}+\varepsilon\right)\right)^{1-m}+C t\right\}^{\frac{1}{1-m}}+\left|D_{\delta_{1}}\right| \mu_{0} \\
& \quad+\left|D_{\delta_{1}}\right|^{1-\frac{1}{p}}\left(\left\|u_{0}\right\|_{L^{p}\left(D_{\delta_{1}}\right)}+\varepsilon\right) \quad \forall 0<\delta_{1}<\delta_{0} / 2, t>0 .
\end{aligned}
$$

Letting first $t \rightarrow 0$ and then $\delta_{1} \rightarrow 0$ in (3.12),

$$
\lim _{t \rightarrow 0} \int_{\Omega_{\delta}}\left|v(x, t)-u_{0}(x)\right| d x=0 \quad \forall 0<\delta<\delta_{0} .
$$

By (3.7), (3.8), (3.13) and Theorem 2.3, $v=u$ in $\left(\bar{\Omega} \backslash\left\{a_{1}, \cdots, a_{i_{0}}\right) \times(0, \infty)\right.$. Hence $u_{j}$ converges to $u$ uniformly in $C^{2,1}\left(\Omega_{\delta} \times\left(t_{1}, t_{2}\right)\right)$ as $j \rightarrow \infty$ for any $0<\delta<\delta_{0}$ and $t_{2}>t_{1}>0$ and the lemma follows.

We next recall two results from [HK2].

Theorem 3.2. (cf. Theorem 1.3 of [HK2]) Suppose that $n \geq 3,0<m<\frac{n-2}{n}$ and $\mu_{0}>0$. Let $\mu_{0} \leq u_{0} \in L_{\text {loc }}^{p}\left(\bar{\Omega} \backslash\left\{a_{1}, \cdots, a_{i_{0}}\right\}\right)$ for some constant $p>\frac{n(1-m)}{2}$ satisfy (1.22) for some constants satisfying (1.18) and $\lambda_{1}, \cdots, \lambda_{i_{0}}, \lambda_{1}^{\prime}, \cdots, \lambda_{i_{0}}^{\prime} \in \mathbb{R}^{+}$. Let $u$ be the solution of (1.23) given by Theorem 2.3 Then

$$
u(x, t) \rightarrow \mu_{0} \quad \text { in } C^{2}(K) \quad \text { as } t \rightarrow \infty
$$

for any compact subset $K$ of $\bar{\Omega} \backslash\left\{a_{1}, \cdots, a_{i 0}\right\}$. 
Theorem 3.3. Suppose that $n \geq 3,0<m<\frac{n-2}{n}$ and $\mu_{0}>0$. Let $\mu_{0} \leq u_{0} \in L_{l o c}^{p}\left(\bar{\Omega} \backslash\left\{a_{1}, \cdots, a_{i_{0}}\right\}\right)$ for some constant $p>\frac{n(1-m)}{2}$ satisfy (1.2) for some constants satisfying

$$
\gamma_{1}>\frac{n-2}{m}, \quad \gamma_{i}>\frac{2}{1-m} \quad \forall i=2, \ldots, i_{0}
$$

and $0<\delta_{1}<\delta_{0}, \lambda_{1}, \cdots, \lambda_{i_{0}} \in \mathbb{R}^{+}$. Let $u$ be the solution of (1.23) given by Theorem 2.3. Then

$$
u(x, t) \rightarrow \infty \quad \text { on } K \quad \text { as } t \rightarrow \infty
$$

for any compact subset $K$ of $\widehat{\Omega}$.

Proof of Theorem 1.4: We will use a modification of the proof of Theorem 1 of [VW2] to construct the oscillating solution $u$ of (1.23) as the limit of a sequence of solutions $u_{j}$ of (1.23) with initial value $u_{0, j}$ that satisfy appropriate blow-up condition at the points $a_{1}, \cdots, a_{i_{0}}$. Let

$$
\alpha_{1}>\frac{n-2}{m}, \quad \alpha_{2}=\frac{\frac{2}{1-m}+n}{2},
$$

and let $K$ be a compact subset of $\widehat{\Omega}$. We choose $j_{1} \in \mathbb{Z}^{+}$such that $j_{1}>\max \left(\delta_{0}^{-1}, \mu_{0}^{1 / \alpha_{1}}, \mu_{0}^{1 / \alpha_{2}}\right)$. Let

$$
u_{0,1}(x)= \begin{cases}j_{1}^{\alpha_{2}} & \forall x \in \bar{\Omega} \backslash \cup_{i=1}^{i_{0}} B_{1 / j_{1}}\left(a_{i}\right) \\ \left|x-a_{i}\right|^{-\alpha_{2}} & \forall x \in B_{1 / j_{1}}\left(a_{i}\right), i=1, \cdots, i_{0} .\end{cases}
$$

Then $u_{0,1}(x) \geq \mu_{0}$ for any $x \in \bar{\Omega} \backslash\left\{a_{1}, \cdots, a_{i_{0}}\right\}$. By Theorem 2.3 there exists a unique solution $u_{1}$ of $(1.23)$ which satisfies $u_{1} \geq \mu_{0}$ in $\left(\bar{\Omega} \backslash\left\{a_{1}, \cdots, a_{i_{0}}\right\}\right) \times(0, \infty)$. By Theorem 3.2 ,

$$
u_{1}(x, t) \rightarrow \mu_{0} \quad \text { in } C^{2}(K) \quad \text { as } t \rightarrow \infty \text {. }
$$

Hence there exists a constant $t_{1}>1$ such that

$$
\mu_{0} \leq u_{1}\left(x, t_{1}\right) \leq \mu_{0}+\frac{1}{2} \quad \forall x \in K
$$

For any $j \in \mathbb{Z}^{+}, j>j_{1}$, let

$$
u_{0,1, j}(x)= \begin{cases}u_{0,1}(x) & \forall x \in \bar{\Omega} \backslash B_{1 / j}\left(a_{1}\right) \\ \left|x-a_{1}\right|^{-\alpha_{1}} & \forall x \in B_{1 / j}\left(a_{1}\right) .\end{cases}
$$

Then $u_{0,1, j}(x) \geq \mu_{0}$ for any $x \in \bar{\Omega} \backslash\left\{a_{1}, \cdots, a_{i_{0}}\right\}$ and $j>j_{1}$. For any $j>j_{1}$, let $u_{2, j}$ be the unique solution of (1.23) with $u_{0}=u_{0,1, j}$ given by Theorem 2.3 which satisfies $u_{2, j} \geq \mu_{0}$ in $\left(\bar{\Omega} \backslash\left\{a_{1}, \cdots, a_{i_{0}}\right\}\right) \times(0, \infty)$. Since $u_{0,1, j}$ converges to $u_{0,1}$ in $L_{l o c}^{p}\left(\bar{\Omega} \backslash\left\{a_{1}, \cdots, a_{i_{0}}\right\}\right)$ as $j \rightarrow \infty$, by Lemma $3.1 u_{2, j}\left(x, t_{1}\right)$ converges to $u_{1}\left(x, t_{1}\right)$ uniformly in $K$ as $j \rightarrow \infty$. Hence there exists $j_{2} \in \mathbb{Z}^{+}, j_{2}>j_{1}$, such that

$$
\left|u_{2, j_{2}}\left(x, t_{1}\right)-u_{1}\left(x, t_{1}\right)\right| \leq \frac{1}{4} \quad \forall x \in K
$$


Let $u_{2}=u_{2, j_{2}}$ and $u_{0,2}=u_{0,1, j_{2}}$. By (3.17) and (3.19),

$$
\mu_{0} \leq u_{2}\left(x, t_{1}\right) \leq \mu_{0}+\frac{3}{4} \quad \forall x \in K
$$

By (3.18) and Theorem 3.3 ,

$$
u_{2}(x, t) \rightarrow \infty \quad \text { in } C^{2}(K) \quad \text { as } t \rightarrow \infty .
$$

Hence there exists a constant $t_{2}>t_{1}+1$ satisfying

$$
u_{2}\left(x, t_{2}\right) \geq 3 \quad \forall x \in K \text {. }
$$

Repeating the above argument we get sequences $\left\{u_{0, k}\right\}_{k=1}^{\infty} \subset L_{l o c}^{p}\left(\bar{\Omega} \backslash\left\{a_{1}, \cdots, a_{i_{0}}\right\}\right),\left\{j_{k}\right\}_{k=1}^{\infty} \subset$ $\mathbb{Z}^{+}$and $\left\{t_{k}\right\}_{k=1}^{\infty} \subset \mathbb{R}^{+}$, such that $j_{k+1}>j_{k}$ and $t_{k+1}>t_{k}+1$ for all $k \in \mathbb{Z}^{+}$, which satisfy

$$
\begin{aligned}
& u_{0, k}(x) \geq \mu_{0} \quad \text { in } \bar{\Omega} \backslash\left\{a_{1}, \cdots, a_{i_{0}}\right\} \quad \forall k \in \mathbb{Z}^{+}, \\
& u_{0, k}(x)=u_{0, k-1}(x) \quad \forall x \in \bar{\Omega} \backslash B_{1 / j_{k}}\left(a_{1}\right), k \geq 2
\end{aligned}
$$

and

$$
u_{0, k}(x)=\left\{\begin{array}{lll}
\left|x-a_{1}\right|^{-\alpha_{2}} & \forall x \in B_{1 / j_{k}}\left(a_{1}\right) & \text { if } k \geq 1 \text { is odd } \\
\left|x-a_{1}\right|^{-\alpha_{1}} & \forall x \in B_{1 / j_{k}}\left(a_{1}\right) & \text { if } k \geq 2 \text { is even }
\end{array}\right.
$$

and if $u_{k}$ is the solution of (1.23) with $u_{0}=u_{0, k}$ given by Theorem 2.3, then $u_{k}$ satisfies

$$
\begin{array}{r}
\mu_{0} \leq u_{k}\left(x, t_{l}\right) \leq \mu_{0}+\frac{1}{2^{l}}+\cdots+\frac{1}{2^{k}} \leq \mu_{0}+\frac{1}{2^{l-1}} \quad \forall x \in K, 1 \leq l \leq k \text { and } l \text { is odd, } \\
u_{k}\left(x, t_{l}\right)>l+\frac{3}{2}-\left(\frac{1}{2^{l}}+\cdots+\frac{1}{2^{k}}\right)>l \quad \forall x \in K, 1 \leq l \leq k \text { and } l \text { is even }
\end{array}
$$

and

$$
\left|u_{k}\left(x, t_{l}\right)-u_{k+1}\left(x, t_{l}\right)\right|<\frac{1}{2^{k}} \quad \forall x \in K, 1 \leq l \leq k, k \in \mathbb{Z}^{+} .
$$

Let

$$
u_{0}(x)= \begin{cases}j_{1}^{\alpha_{2}} & \forall x \in \bar{\Omega} \backslash \cup_{i=1}^{i_{0}} B_{1 / j_{1}}\left(a_{i}\right) \\ \left|x-a_{i}\right|^{-\alpha_{2}} & \forall x \in B_{1 / j_{1}}\left(a_{i}\right), i=2, \cdots, i_{0} \\ u_{0, k}(x) & \forall 1 / j_{k+1} \leq\left|x-a_{1}\right| \leq 1 / j_{k}, k \geq \mathbb{Z}^{+} .\end{cases}
$$

Then $u_{0} \geq \mu_{0}$ in $\bar{\Omega} \backslash\left\{a_{1}, \cdots, a_{i_{0}}\right\}$,

$$
u_{0}(x)= \begin{cases}\left|x-a_{1}\right|^{-\alpha_{2}} & \forall 1 / j_{k+1} \leq\left|x-a_{1}\right| \leq 1 / j_{k}, k \geq \mathbb{Z}^{+} \quad \text { and } k \text { is odd } \\ \left|x-a_{1}\right|^{-\alpha_{1}} & \forall 1 / j_{k+1} \leq\left|x-a_{1}\right| \leq 1 / j_{k}, k \geq \mathbb{Z}^{+} \quad \text { and } k \text { is even }\end{cases}
$$

and $u_{0, k}$ converges to $u_{0}$ in $L_{l o c}^{p}\left(\bar{\Omega} \backslash\left\{a_{1}, \cdots, a_{i_{0}}\right\}\right)$ as $k \rightarrow \infty$. let $u$ be the unique solution of (1.23) given by Theorem 2.3. Then by Lemma $3.1 u_{k}$ converges to $u$ on every compact subset of $\widehat{\Omega} \times(0, \infty)$ as $k \rightarrow \infty$. letting $k \rightarrow \infty$ in (3.20) and (3.21) we have

$$
\begin{aligned}
& \mu_{0} \leq u\left(x, t_{l}\right) \leq \mu_{0}+\frac{1}{2^{l-1}} \quad \forall x \in K, l \in \mathbb{Z}^{+} \text {and } l \text { is odd } \\
\Rightarrow & \lim _{k \rightarrow \infty} u\left(x, t_{2 k-1}\right)=\mu_{0} \quad \text { uniformly in } K
\end{aligned}
$$


and

$$
\begin{aligned}
& u\left(x, t_{l}\right) \geq l \quad \forall x \in K, l \in \mathbb{Z}^{+} \text {and } l \text { is even } \\
\Rightarrow \quad & \lim _{k \rightarrow \infty} u\left(x, t_{2 k}\right)=\infty \quad \text { uniformly in } K
\end{aligned}
$$

and the theorem follows.

\section{References}

[A] D.G. Aronson, The porous medium equation, CIME Lectures in Some problems in Nonlinear Diffusion, Lecture Notes in Mathematics 1224, Springer-Verlag, New York, 1986.

[CL] B. Choi and K. Lee, Multi-D fast diffusion equation via diffusive scaling of generalized Carleman kinetic equation, arXiv:1510.08997.

[DaK] B.E.J. Dahlberg and C. Kenig, Non-negative solutions of generalized porous medium equations, Revista Matemática Iberoamericana 2 (1986), 267-305.

[DK] P. Daskalopoulos and C.E. Kenig, Degenerate diffusion-initial value problems and local regularity theory, Tracts in Mathematics 1, European Mathematical Society, 2007.

[DS1] P. Daskalopoulos and N. Sesum, On the extinction profile of solutions to fast diffusion, J. Reine Angew. Math. 622 (2008), 95-119.

[DS2] P. Daskalopoulos and N. Sesum, The classification of locally conformally flat Yamabe solitons, Advances in Math. 240 (2013), 346-369.

[GS] F. Golse and F. Salvarani, The nonlinear diffusion limit for generalized Carleman models: the initial-boundary value problem, Nonlinearity 20 (2007), 927-942.

[HK1] K.M. Hui and S. Kim, Existence of Neumann and singular solutions of the fast diffusion equation, Discrete Contin. Dyn. Syst. Series-A 35 (2015), no. 10, 4859-4887.

[HK2] K.M. Hui and S. Kim, Existence and large time behaviour of finite points blow-up solutions of the fast diffusion equation, arXiv:1712.05515v2.

[HKs] K.M. Hui and Soojung Kim, Asymptotic large time behaviour of singular solutions of the fast diffusion equation, Discrete Contin. Dyn. Syst. Series-A 37 (2017), no. 11, 5943-5977.

[HP] M.A. Herrero and M. Pierre, The Cauchy problem for $u_{t}=\Delta u^{m}$ when $0<m<1$, Transactions A. M. S. 291 (1985), no. 1, 145-158.

[HPW] F. Huang, R. Pan and Z. Wang, $L^{1}$ convergence to the Barenblatt solution for compressibe Euler equations with damping, Arch. Ration. Mech. Anal. 200 (2011), no. 2, 665-689. 
[Hs] S.Y. Hsu, Existence and asymptotic behaviour of solutions of the very fast diffusion equation, Manuscripta Math. 140 (2013), no. 3-4, 441-460.

[K] T. Kato, Schrödinger operators with singular potentials, Israel J. Math. 13 (1973), 135-148.

[LSU] O.A. Ladyzenskaya, V.A. Solonnikov and N.N. Uraltceva, Linear and quasilinear equations of parabolic type, Transl. Math. Mono. vol. 23, Amer. Math. Soc., Providence, R.I., U.S.A., 1968.

[LZ] T. Luo and H. Zeng, Global existence and smooth solutions and convergence to Barenblatt solutions for the physical vacuum free boundary problem of compressible Euler equations with damping, Commun. on Pure and Applied Math. 69 (2016), no. 7, 1354-1396.

[PS] M. del Pino and M. Sáez, On the extinction profile for solutions of $u_{t}=\Delta u^{(N-2) /(N+2) \text {, }}$ Indiana Univ. Math. J. 50 (2001), no. 1, 611-628.

[PV] A.de Pablo and J.L. Vazquez, Travelling waves and finite propagation in a reactiondiffusion equation, J. Differential Equations 93 (1991), 19-61.

[V1] J.L. Vazquez, Smoothing and Decay Estimates for Nonlinear Diffusion Equations, Oxford Lecture Series in Mathematics and its Applications 33, Oxford University Press, Oxford, 2006.

[V2] J.L. Vazquez, The porous medium equation. Mathematical theory. Oxford Mathematical Monographs. The Clarendon Press, Oxford University Press, Oxford, 2007. xxii+624 pp.

[VW1] J.L. Vazquez and M. Winkler, The evolution of singularities in fast diffusion equations: infinite-time blow-down, SIAM J. Math. Anal. 43 (2011), no. 4, 1499-1535.

[VW2] J.L. Vazquez and M. Winkler, Highly time-oscillating solutions for very fast diffusion equations. J. Evol. Equ. 11 (2011), no. 3, 725-742. 